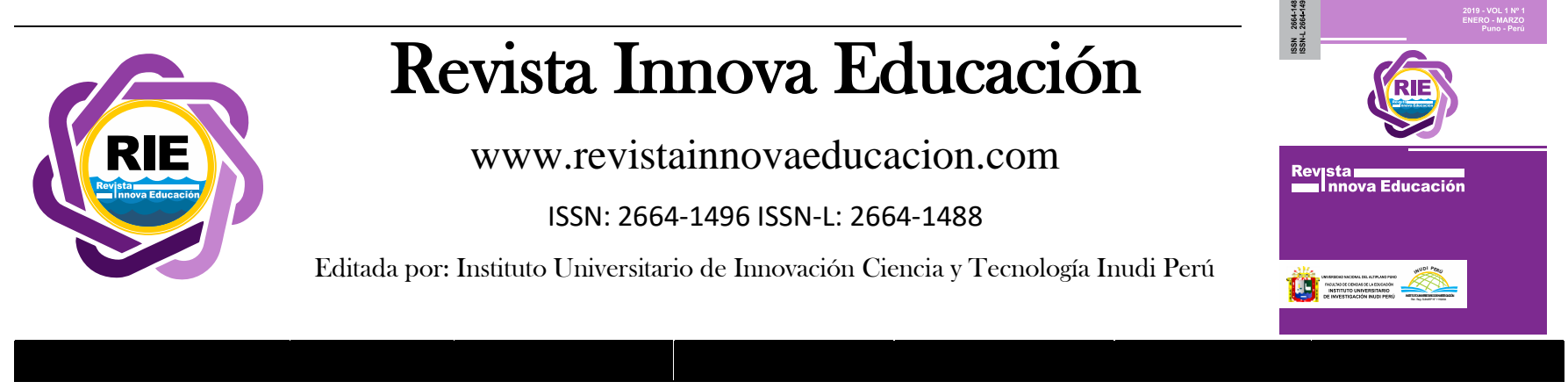

\title{
Estilos de aprendizaje y los desempeños académicos del área de comunicación en los estudiantes de educación básica
}

\author{
Learning styles and academic performance of the communication area in the basic education \\ students
}

\author{
Marisol Yana ${ }^{1}$ (D); Magna Mamani ${ }^{1}, \operatorname{Luz~Cusi}^{1}$ (i), Hector Adco ${ }^{1}$
}

DOI: https://doi.org/10.35622/j.rie.2019.01.004

${ }^{1}$ Universidad Nacional del Altiplano, Escuela Profesional de Educación Secundaria, Av. Floral No 1153, Ciudad Universitaria,
Puno, Perú. marisolyana@ @unap.edu.pe

Recibido el 21/01/2019/ Aceptado el 27/01/2019

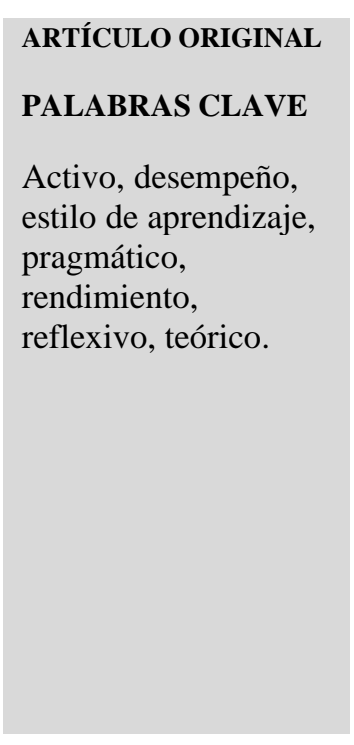

\section{KEYWORDS}

Active, performance, learning style, pragmatic, performance, reflective, theoretical.
La investigación consistió en determinar la relación entre estilos de aprendizaje y el desempeño académico en el área de Comunicación en estudiantes de la institución educativa "Carlos Rubina Burgos" de Puno, 2017. Fue de tipo básico, con diseño no experimental transeccional descriptivo - correlacional. 63 estudiantes constituyeron la muestra censal. Se utilizó las técnicas: encuesta y la observación sistemática indirecta, el instrumento fue el Cuestionario Honey-Alonso de Estilos de Aprendizaje (CHAEA) consistente en 80 ítems dividido en cuatro secciones de 20 ítems correspondientes a estilos de aprendizaje (activo, reflexivo, teórico y pragmático) y una ficha para determinar el nivel de desempeño académico en el área de Comunicación. Para analizar los datos se empleó el (SPSS) versión 22 y la correlación de Pearson, se determinó que entre el estilo de aprendizaje reflexivo y el nivel de desempeño académico la correlación es positiva moderada $\left(\mathrm{r}=, 489^{* *}\right)$. Por otra parte, la relación existente entre los estilos de aprendizaje teórico $\left(\mathrm{r}=, 353^{* *}\right)$ y pragmático $(\mathrm{r}=, 292 *)$ con el desempeño académico en el área de comunicación es positiva baja y significativa y en el estilo de aprendizaje activo y el desempeño académico existe una relación positiva muy baja $(\mathrm{r}=.189)$ y no significativa. En cuanto al desempeño académico se encuentran en el nivel logro esperado en un $61.9 \%$.

The research consisted of determining the relationship between learning styles and academic performance in the area of Communication in students of the educational institution "Carlos Rubina Burgos" of Puno, 2017. It was of a basic type, with nonexperimental descriptive-correlational transectional design. 63 students constituted the census sample. We used the techniques: survey and indirect systematic observation, the instrument was the Honey-Alonso Learning Styles Questionnaire (CHAEA) consisting of 80 items divided into four sections of 20 items corresponding to learning styles (active, reflective, theoretical and pragmatic) and a file to determine the level of academic performance in the area of Communication. To analyze the data we used the (SPSS) version 22 and the Pearson correlation, it was determined that between the reflective learning style and the level of academic performance the correlation is 
moderate positive $(\mathrm{r}=, 489 * *)$. On the other hand, the relationship between the theoretical $(\mathrm{r}=, 353 * *)$ and pragmatic $(\mathrm{r}=, 292 *)$ learning styles with academic performance in the communication area is low and significant positive and in the learning style Active and academic performance there is a very low positive relationship $(\mathrm{r}=.189)$ and not significant. In terms of academic performance, they are at the expected level of achievement of $61.9 \%$.

\section{INTRODUCCIÓN}

Cada vez se plantean retos complejos al estudiante, como: Generar filtros cognitivos ante la saturación informativa; desarrollar procedimientos de gestión del conocimiento, ya que los avances científico-tecnológicos exigen un permanente aprendizaje; y finalmente, alcanzar una alfabetización gráfica para estar en la capacidad de entender, comprender y ser críticos frente a las nuevas formas de acceso a la información, (Pozo, Monereo, \& Castelló, 2001). Es necesario que el estudiante movilice, regule y utilice sus propias estrategias, metodologías, procedimientos, tiempos, recursos,... para aprender; por lo que corresponde al docente: orientar, mediar, apoyar y guiar este proceso mediante las actividades de aprendizajes de los estudiantes (Biggs, 2006). Actualmente, la actividad pedagógica viene demostrando su intención constante de perfeccionamiento y el gradual conocimiento de la diversidad humana, que se manifiesta en la implementación de prácticas y enfoques que rehúyen de la homogeneidad y uniformidad, aproximándose más a la heterogeneidad y diversidad (Farias, Díaz, \& Miranda, 2012). Actividad pedagógica que depende de los estilos de aprendizaje que uno elige. Es que es determinante el estilo de aprendizaje para lograr con éxito los desempeños académicos en los estudiantes.

\section{Estilos de aprendizaje}

El estilo es un conjunto de aptitudes, preferencias, tendencias y actitudes que tiene una persona para hacer algo y que se manifiesta a través de un patrón conductual y de distintas destrezas que lo distingue de las demás (García, Santizo, \& Alonso, 2009; Rojas, Salas, \& Jimenez, 2006). Y el aprendizaje es la acción de aprender algún arte u oficio; por otra parte, aprender es adquirir el conocimiento de alguna cosa por medio del estudio o la experiencia (Aragon de Viau, 2000). Por su lado, Garay (2015), afirma, el aprendizaje es simplemente el estilo cognitivo manifestado por el individuo frente a una tarea de aprendizaje y refleja sus estrategias preferidas, habituales y naturales para aprender. A su vez, Cachay (2014), explica que son comportamientos distintivos que indican cómo la persona aprende y se adapta a su ambiente. Por su lado, Jara (2010),el aprendizaje es un proceso de adquisición de conocimientos teóricos y prácticos, en el que intervienen diferentes factores como: la motivación, atención, aptitudes, habilidades, memoria, inteligencia y la realidad educativa en donde se produce el cambio en el estudiante. Los alumnos deben "aprender a aprender" y "los profesores deben reconocer las diferencias individuales de sus alumnos para personalizar su educación tratando de que sus preferencias en cuanto a los estilos de enseñanza no influyan en los estilos de aprendizaje de los Alumnos". Además, los profesores enseñan como les gustaría aprender las cosas (C. Alonso, Gallego, \& Honey, 1994; García Cue, 2006). De tal manera que, el estilo de enseñar preferido por el profesor, muy influido por su estilo de aprender, puede significar un favoritismo inconsciente para los estudiantes con el mismo estilo de aprendizaje, los mismos sitemas de pensamiento . Así mismo, el analisis de los estilos de aprendizaje ofrece indicadores que ayudan a guiar las interacciones de la persona con las realidades exitenciales, facilitando un camino de auto y heteroconocimiento Gallego \& Alonso (2007), siendo así, la apariencia externa de las habilidades de mediación de un individuo (Gregorc, 1982).

Dunn \& Dunn (1984); Esteban \& Ruiz (1996); Rettis (2016), coinciden relativamente al afirman que el estilo de aprendizaje consiste en estrategias y formas particulares de comportarse, responder y utilizar los estímulos en un contexto de aprendizaje concentrándose, tratándolo y reteniendo la información nueva. 
Estilos de aprendizaje que indican el significado natural por el cual la persona fácil, efectiva y eficientemente se comprende a sí misma, al mundo y la relación entre ambos (Catalina Alonso, Gallego, \& Honey, 2007). Sostienen también que es la forma distintiva y característica que tiene el estudiante para acercarse a un proyecto o una situación de aprendizaje, independientemente de si incluye la decisión explícita o implícita del estudiante. Incluso, algunas capacidades de aprender que se destaca por encima de otras como resultado del aparato hereditario de las experiencias vitales propias, y de las exigencias del medio ambiente actual son estilos de aprendizaje (Coloma, Manrique, Revilla, \& Tafur, 2008).

Una definición más clara y ajustada al ámbito educativo corresponde a Keefe \& Ferrel (1990) al plantear que los estilos de aprendizaje son los rasgos cognitivos, afectivos y fisiológicos, que sirven como guías para ver cómo los estudiantes responden a sus contextos de aprendizajes. A todo lo mencionado, el estilo de aprendizaje que predomina en el aula será una herramienta para el docente para adaptar el estilo de enseñanza y lograr el mejor desempeño académico en los estudiantes (Gutiérrez, 2018).

Catalina Alonso et al. (2007) expresan que para Honey y Mumford los estilos de aprendizaje son los siguientes:

\section{a. Activo}

Las personas con este estilo de aprendizaje se involucran plenamente y sin prejuicios en experiencias nuevas. Tienen una mente abierta, son nada escépticas y realizan con entusiasmo las actividades nuevas. Son personas que viven el presente y llenos de actividad. Piensan que por lo menos una vez hay que intentarlo todo. Tan pronto como disminuye la emoción de una actividad, se aproxima a otra. Se crecen ante los desafíos que suponen experiencias nuevas y se aburren los plazos largos. Son personas que se sienten a gusto en grupo, se implican en los asuntos de los otros y centran alrededor todas las actividades.

\section{b. Reflexivo}

Los reflexivos aprenden también con las nuevas experiencias pero no les gusta estar directamente implicados en ellas. Reúnen datos, analizándolos con detenimiento antes de llegar a alguna conclusión. Disfrutan observando la actuación de los demás, escuchándoles pero no intervienen hasta que se han adueñado de la situación.

\section{c. Teórico}

Las personas con este estilo adaptan e integran las observaciones dentro de teorías lógicas y complejas. Enfocan los problemas de forma vertical escalonada, por etapas lógicas. Suelen ser perfeccionistas. Les gusta analizar y sintetizar. Son profundos en su sistema de pensamiento, a la hora de establecer principios, teorías y modelos. Para ellos, si es lógico es bueno. Buscan la racionalidad y la objetividad, alejándose de lo subjetivo y ambiguo.

\section{d. Pragmático}

Las personas con este estilo son hábiles en la aplicación práctica de las ideas. Descubren el aspecto positivo de las nuevas ideas y aprovechan la primera oportunidad para experimentarlas. Les gusta actuar rápidamente y con seguridad con los proyectos que les atraen. Tienden a ser impacientes cuando hay personas que teorizan. Pisan tierra cuando hay que tomar una decisión o resolver un problema.

Algunos estudios dan evidencia de la existencia de diferencias significativas tales como: Manzano \& Hidalgo (2009), concluyen que, los estilos pragmático y reflexivo se asocian al uso frecuente de estrategias de lectura; y estos influyen en el rendimiento académico de la lengua extranjera. Por otro lado, 
García, Sanchez, Gutierrez, \& Jimenez (2012) indican que existe un leve incremento en las puntuaciones de estilos de aprendizaje después de aplicar estrategias de aprendizaje en los curso de postgrado. (Cachay, 2014), señala que el valor del coeficiente de correlación que existe entre los estilos de aprendizaje y el rendimiento académico es de 50,3, indicando así que existe una correlación positiva y significativa. En su investigación, Jara (2010), llegó a la conclusión de que existe una correlación significativa entre los estilos de aprendizaje reflexivo y teórico con el rendimiento académico en Educación para el trabajo. También, encontramos que el curso en que se encuentran los alumnos influye en sus estilos de aprendizaje, la edad parece que varían los estilos de aprendizaje de los alumnos y finalmente la diferencia de sexo en los alumnos influye en sus estilos de aprendizaje (Catalina Alonso, 1992).

\section{Desempeños académicos}

El desempeño académico está dado por el promedio de las calificativos obtenidos por los estudiantes en los diferentes espacios académicos cursados (Parra, Cerda, López, \& Saiz, 2014). Se debe utilizar estrategias comportamentales adecuadas a fin de lograr un mejor desempeño académico. En este sentido, los hábitos de estudio constituyen una estrategia de aprendizaje relevante que pueden ayudar a los alumnos a mejorar su rendimiento académico (Gaeta, Cavazos, \& Arroyo, 2016; Suárez et al., 2012) . Diversos estudios muestran que cuando un alumno se siente competente para autorregular su aprendizaje incrementa su motivación hacia el estudio, así como su rendimiento académico (Rosário et al., 2009; Zimmerman, 1989). El logro de un buen desempeño académico se considera como un proceso complejo y dependiente principalmente de factores personales y del contexto de aprendizaje, incluso el tiempo es determinante (De Murcia et al., 2013; Gaeta, Martha Leticia; Teruel, M. Pilar; Orejudo, 2012; Yana \& Adco, 2018).

Rettis (2016), el rendimiento académico es la medida de la capacidad correspondiente o indicativa que muestra el estudiante, en forma estimativa, respecto de sus aprendizajes, como resultado de un proceso de instrucción o formación. Para Jimenez (2000), es el nivel de conocimientos demostrado en un área o materia comparado con la norma de edad y nivel académico (Diaz, 2010). Es un sistema técnico pedagógico que mide los logros y la construcción de conocimientos en los estudiantes, los cuales se generan a atravéz de la intervención de didácticas educativas que son evaluadas a mediantes los métodos cualitativos y cuantitativos en una materia (Cachay, 2014; Rettis Salazar, 2016).

\section{MÉTODO Y MATERIALES}

La investigación se localizó en la Institución Educativa Secundaria "Carlos Rubina Burgos" del distrito, provincia y región de Puno - Perú. El periodo de duración de esta investigación corresponde al I trimestre del año escolar 2017. Para obtener los datos y recopilar la información requerida para cada variable de la esta investigación, se ha empleado dos técnicas, las cuales son: $\boldsymbol{a}$ ) La Encuesta

técnica de investigación social sirve para indagar, explorar y recolectar datos a través de preguntas formuladas de forma directa o indirecta a los sujetos que constituyen las unidades de análisis del estudio investigativo (Carrasco, 2005). En la investigación se utilizó para recolectar los datos correspondientes a la variable "Estilos de aprendizaje". En este caso, las preguntas fueron formuladas indirectamente por medio del Cuestionario Honey - Alonso de Estilos de Aprendizaje (CHAEA). El cuestionario consta de una ficha de datos socio-académicos, un cuadernillo de 80 ítems dividido en cuatro secciones de 20 ítems correspondientes a los cuatro estilos de aprendizaje (Activo, Reflexivo, Teórico y Pragmático). b) $\mathbf{L a}$ Observación Sistemática Indirecta, es un proceso sistemático de obtención, recopilación y registro de datos empíricos de un objeto, un suceso, un acontecimiento o conducta humana con el propósito de procesarlo y transformarlo en información (Carrasco, 2005). Dicho proceso puede ser directo o indirecto. En la investigación se empleó esta técnica para recopilar la información contenida en documentos (Reporte de Notas Registradas - Trimestre I - 2017) estrechamente relacionado con la variable "Desempeño académico 
en el área de Comunicación". Se observó indirectamente, ya que se recurrió a unos documentos para cerciorarse de las notas obtenidas por las estudiantes. $\boldsymbol{c}$. Confiabilidad y Validez. el cuestionario Honey Alonso de Estilos de Aprendizaje (CHAEA), en el año 1992, Catalina Alonso diseñó y realizó una investigación con variedad de pruebas estadísticas sobre una muestra de 1371 estudiantes de las Universidades Complutense y Politécnica de Madrid. Para la validez, efectuó las siguientes pruebas estadísticas: Análisis de contenidos e ítems, Análisis factorial de los 80 ítems, Análisis factorial de los 20 ítems de cada estilo y de los cuatro estilos partiendo de las medias totales de sus 20 ítems. Los valores obtenidos para cada estilo fueron: 0.84925 para el teórico, 0.82167 para el reflexivo, 0.78633 para el pragmático y 0.74578 para el activo y aplicó la prueba Alfa de Cronbach, alcanzando así valores para cada estilo de aprendizaje.

\section{RESULTADOS}

Tabla 1 Confiabilidad de la estructura interna del Cuestionario Honey-Alonso de Estilos de Aprendizaje (CHAEA)

\begin{tabular}{ccccc}
\hline & \multicolumn{4}{c}{ Estilos de Aprendizaje } \\
\cline { 2 - 5 } & Activo & Reflexivo & Teórico & Pragmático \\
\hline Alfa de Cronbach & 0.6272 & 0.7275 & 0.6584 & 0.5854 \\
\hline
\end{tabular}

Fuente: Alonso, C., Gallego, D., y Honey, P. (2007). Los Estilos de Aprendizaje. Procedimientos de diagnóstico y mejora (Séptima ed.). Bilbao: Mensajero.

Tabla 2 Estadísticos descriptivos de los estilos de aprendizaje

\begin{tabular}{lcccc}
\hline Estadísticos & Activo & Reflexivo & Teórico & Pragmático \\
\hline $\mathrm{N}^{\circ}$ de casos & 63 & 63 & 63 & 63 \\
Media & 10.73 & 13.67 & 13.14 & 12.32 \\
Desviación estándar & 2.936 & 2.410 & 2.614 & 2.520 \\
Coef. de variabilidad & $27.4 \%$ & $17.6 \%$ & $19.9 \%$ & $20.5 \%$ \\
Varianza & 8.620 & 5.806 & 6.834 & 6.349 \\
Mínimo & 6 & 8 & 4 & 6 \\
Máximo & 17 & 18 & 18 & 16 \\
\hline
\end{tabular}

Fuente: Cuestionario de Honey - Alonso de Estilos de Aprendizaje (CHAEA)

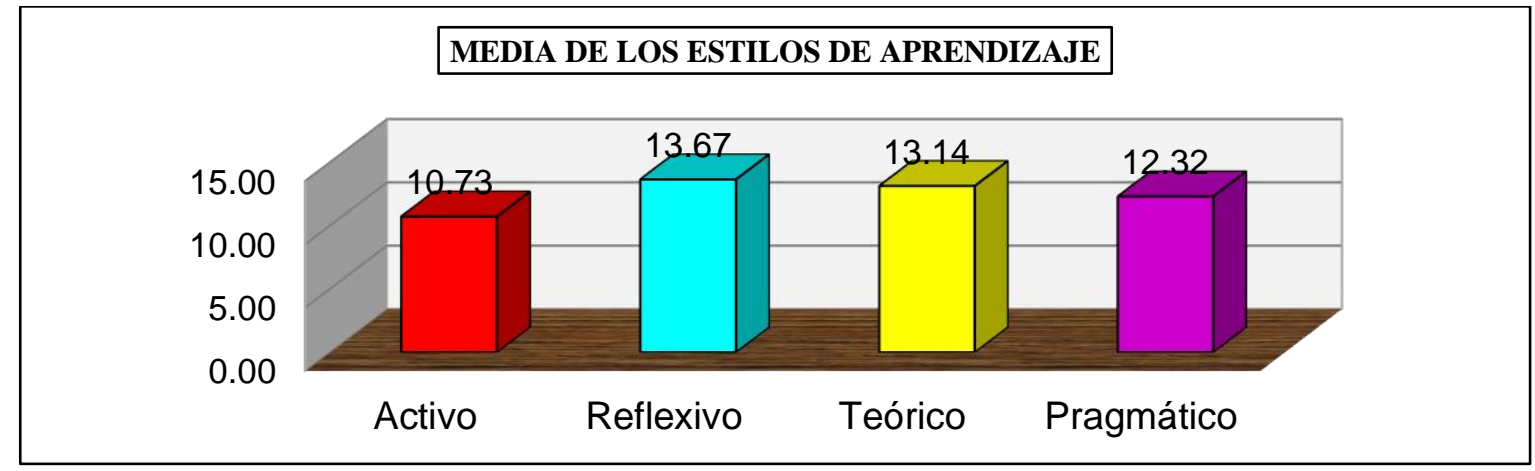

Figura 1. Distribución de la media de los estilos de aprendizaje

En la tabla 2 y figura 1 se presenta los resultados respecto a los estilos de aprendizaje correspondiente a las estudiantes. En $1^{\circ}$ lugar se ubica el estilo de aprendizaje reflexivo, con un 13.67 puntos y una desviación estándar de 2.410; dentro de un rango que va desde 8 puntos como mínimo hasta 18, como máximo. El coeficiente de variabilidad se da en $17.6 \%$, lo cual indica que sus datos son regularmente variables. En $2^{\circ}$ lugar aparece el estilo de aprendizaje teórico, con un 13.14 puntos y una desviación estándar de 2.614; dentro de un rango que va desde 4 puntos como mínimo hasta 18, como máximo. Así también, posee un 
coeficiente de variabilidad de $19.9 \%$, lo cual explica que sus datos son regularmente variables. En $3^{\circ}$ lugar está el estilo de aprendizaje pragmático, con 12.32 puntos y una desviación estándar de 2.520; dentro de un rango que va desde 6 puntos como mínimo hasta 16, como máximo. Asimismo, presenta un coeficiente de variabilidad de $20.5 \%$, lo cual afirma que sus datos son variables. El estilo de aprendizaje activo se sitúa en el $4^{\circ}$ lugar por conseguir una media de 10.73 puntos y una desviación estándar de 2.936; dentro de un rango que va desde 6 puntos como mínimo hasta 17, como máximo. Además, su coeficiente de variabilidad de $27.4 \%$ permite aseverar que sus datos son muy variables. Por lo tanto, cabe reafirmar que el estilo de aprendizaje reflexivo (13.67) predomina en las estudiantes del $4^{\circ}$ grado, lo cual implica que ellas se desenvuelven mejor cuando se trata de ser: ponderadas, improvisadoras, descubridoras, arriesgadas o espontáneas. El que menos resalta es el estilo de aprendizaje activo (10.73), por lo que se infiere que presentan ciertas dificultades cuando se trata de ser: animadoras, improvisadoras, descubridoras, arriesgadas o espontáneas.

Tabla 3 Niveles de preferencia respecto del estilo de aprendizaje activo

\begin{tabular}{|c|c|c|c|c|c|c|c|c|c|c|c|c|}
\hline \multirow{3}{*}{$\begin{array}{c}\text { Estilo de } \\
\text { Aprendizaje }\end{array}$} & \multicolumn{10}{|c|}{ Niveles de Preferencia } & \multirow{2}{*}{\multicolumn{2}{|c|}{ Total }} \\
\hline & \multicolumn{2}{|c|}{$\begin{array}{c}\text { Muy Baja } \\
(0-6)\end{array}$} & \multicolumn{2}{|c|}{$\begin{array}{c}\text { Baja } \\
(7-8)\end{array}$} & \multicolumn{2}{|c|}{$\begin{array}{l}\text { Moderada } \\
(9-12)\end{array}$} & \multicolumn{2}{|c|}{$\begin{array}{c}\text { Alta } \\
(13-14)\end{array}$} & \multicolumn{2}{|c|}{$\begin{array}{c}\text { Muy Alta } \\
(15-20)\end{array}$} & & \\
\hline & fi & $\%$ & fi & $\%$ & fi & $\%$ & fi & $\%$ & fi & $\%$ & fi & $\%$ \\
\hline Activo & 4 & 6.3 & 13 & 20.6 & 27 & 42.9 & 11 & 17.5 & 8 & 12.7 & 63 & 100 \\
\hline
\end{tabular}

Fuente: Cuestionario de Honey - Alonso de Estilos de Aprendizaje (CHAEA)

Tabla 4 Niveles de preferencia respecto del estilo de aprendizaje reflexivo

\begin{tabular}{|c|c|c|c|c|c|c|c|c|c|c|c|c|}
\hline \multirow{3}{*}{$\begin{array}{l}\text { Estilo de } \\
\text { Aprendizaje }\end{array}$} & \multicolumn{10}{|c|}{ Niveles de Preferencia } & \multirow{2}{*}{\multicolumn{2}{|c|}{ Total }} \\
\hline & \multicolumn{2}{|c|}{$\begin{array}{c}\text { Muy Baja } \\
(0-10)\end{array}$} & \multicolumn{2}{|c|}{$\begin{array}{c}\text { Baja } \\
(11-13)\end{array}$} & \multicolumn{2}{|c|}{$\begin{array}{c}\text { Moderada } \\
(14-17)\end{array}$} & \multicolumn{2}{|c|}{$\begin{array}{c}\text { Alta } \\
(18-19)\end{array}$} & \multicolumn{2}{|c|}{$\begin{array}{l}\text { Muy Alta } \\
\text { (20) }\end{array}$} & & \\
\hline & fi & $\%$ & fi & $\%$ & fi & $\%$ & fi & $\%$ & fi & $\%$ & fi & $\%$ \\
\hline Reflexivo & 8 & 12.7 & 18 & 28.6 & 36 & 57.1 & 1 & 1.6 & 0 & 0.0 & 63 & 100 \\
\hline
\end{tabular}

Fuente: Cuestionario de Honey - Alonso de Estilos de Aprendizaje (CHAEA)

Tabla 5 Niveles de preferencia respecto del estilo de aprendizaje teórico

\begin{tabular}{|c|c|c|c|c|c|c|c|c|c|c|c|c|}
\hline \multirow{3}{*}{$\begin{array}{l}\text { Estilo de } \\
\text { Aprendizaje }\end{array}$} & \multicolumn{10}{|c|}{ Niveles de Preferencia } & \multirow{2}{*}{\multicolumn{2}{|c|}{ Total }} \\
\hline & \multicolumn{2}{|c|}{$\begin{array}{c}\text { Muy Baja } \\
(0-6)\end{array}$} & \multicolumn{2}{|c|}{$\begin{array}{c}\text { Baja } \\
(7-9)\end{array}$} & \multicolumn{2}{|c|}{$\begin{array}{c}\text { Moderada } \\
(10-13)\end{array}$} & \multicolumn{2}{|c|}{$\begin{array}{c}\text { Alta } \\
(14-15)\end{array}$} & \multicolumn{2}{|c|}{$\begin{array}{l}\text { Muy Alta } \\
(16-20)\end{array}$} & & \\
\hline & fi & $\%$ & fi & $\%$ & fi & $\%$ & $\mathbf{f i}$ & $\%$ & fi & $\%$ & fi & $\%$ \\
\hline Teórico & 1 & 1.6 & 7 & 11.1 & 21 & 33.3 & 26 & 41.3 & 8 & 12.7 & 63 & 100 \\
\hline
\end{tabular}

Fuente: Cuestionario de Honey - Alonso de Estilos de Aprendizaje (CHAEA)

$\underline{\text { Tabla } 6}$ Niveles de preferencia respecto del estilo de aprendizaje pragmático

\begin{tabular}{|c|c|c|c|c|c|c|c|c|c|c|c|c|}
\hline \multirow{3}{*}{$\begin{array}{l}\text { Estilo de } \\
\text { Aprendizaje }\end{array}$} & \multicolumn{10}{|c|}{ Niveles de Preferencia } & \multirow{2}{*}{\multicolumn{2}{|c|}{ Total }} \\
\hline & \multicolumn{2}{|c|}{$\begin{array}{c}\text { Muy Baja } \\
(0-8)\end{array}$} & \multicolumn{2}{|c|}{$\begin{array}{c}\text { Baja } \\
(9-10)\end{array}$} & \multicolumn{2}{|c|}{$\begin{array}{c}\text { Moderada } \\
(11-13)\end{array}$} & \multicolumn{2}{|c|}{$\begin{array}{c}\text { Alta } \\
(14-15) \\
\end{array}$} & \multicolumn{2}{|c|}{$\begin{array}{c}\text { Muy Alta } \\
(16-20)\end{array}$} & & \\
\hline & fi & $\%$ & fi & $\%$ & fi & $\%$ & fi & $\%$ & fi & $\%$ & fi & $\%$ \\
\hline Pragmático & 5 & 7.9 & 8 & 12.7 & 26 & 41.3 & 18 & 28.6 & 6 & 9.5 & 63 & 100 \\
\hline
\end{tabular}

Fuente: Cuestionario de Honey - Alonso de Estilos de Aprendizaje (CHAEA)

En la tabla 3 se presenta los resultados respecto del estilo de aprendizaje activo. Así de esta forma, se puede observar que del $100 \%$ (63) de estudiantes, el $42.9 \%$ evidencian un nivel de preferencia moderada; el $20.6 \%$, una preferencia baja; el $17.5 \%$, una preferencia alta; el $12.7 \%$, una preferencia muy alta frente a un $6.3 \%$ de estudiantes que evidencian una preferencia muy baja. Estos resultados indican que la mayoría 
(42.9\%) de las estudiantes se caracterizan de forma moderada por ser: animadoras, improvisadoras, descubridoras, arriesgadas y espontáneas. Es decir, resaltan por su cualidad de poseer una mente abierta y no ser escépticas. Por ello generalmente se embarcan en experiencias diferentes y nuevas, con tal de disfrutar y vivir el presente. Tan pronto como disminuye la emoción, se aproximan a otras más novedosas, porque eso representa para ellas un continuo desafío. No les gusta planificar o tener que cumplir los plazos. Prefieren actuar espontáneamente, estar en grupo, compartir sus emociones e implicarse en los asuntos de los otros.

En la tabla 4 se muestra los resultados respecto del estilo de aprendizaje reflexivo. Así pues, se aprecia que del 100\% (63) de estudiantes, el 57.1\% indican una preferencia moderada; el 28.6\%, una preferencia baja; el $12.7 \%$, una preferencia muy baja, el $1.6 \%$, una preferencia alta y ninguna $(0.0 \%)$ estudiante tiene el nivel de preferencia muy alta. A partir de estos resultados, es posible señalar que la mayoría (57.1\%) de las estudiantes se caracterizan de forma moderada por ser: ponderadas, concienzudas, receptivas, analíticas y exhaustivas. En su actividad de aprendizaje, consideran las experiencias propias o ajenas para observarla desde distintas perspectivas, analizarla exhaustivamente, para luego de forma prudente sacar su conclusión o tomar una decisión. En cuanto se hayan adueñado de la situación, escuchado y analizado las ideas, recién intervienen con su opinión.

En la tabla 5 se expone los resultados respecto del estilo de aprendizaje teórico. De esta forma, se demuestra que del 100\% (63) de estudiantes, el 41.3\% precisan tener una preferencia alta; el 33.3\%, una preferencia moderada; el $12.7 \%$, una preferencia muy alta; en cambio, el $11.1 \%$ y $1.6 \%$ de estudiantes evidencian los niveles de preferencia baja y muy baja, respectivamente. Estos resultados permiten aseverar que la mayoría (41.3\%) tiene preferencia alta cuando se trata de ser: metódicas, lógicas, objetivas, críticas y estructuradas. En su actividad de aprendizaje, resaltan por su cualidad de adaptar e integrar sus observaciones dentro de teorías lógicas y complejas. Analizan y ven los problemas de forma vertical escalonada, por etapas lógicas.

En la tabla 6 se da a conocer los resultados respecto del estilo de aprendizaje pragmático. Así es posible señalar que del 100\% (63) de estudiantes, el $41.3 \%$ manifiestan una preferencia moderada; el 28.6\%, una preferencia alta; el $12.7 \%$, una preferencia baja; el $9.5 \%$, una preferencia muy alta y el $7.9 \%$, una preferencia muy baja. Partiendo de estos resultados, se puede indicar que la mayoría $(41.3 \%)$ de las estudiantes tienen una preferencia moderada cuando se trata de ser: experimentadoras, prácticas, directas, eficaces y realistas. Las estudiantes con este estilo, son hábiles en la aplicación práctica de las ideas. Descubren el aspecto positivo de las ideas novedosas y en seguida se orienta a experimentarlas. Les gusta actuar rápidamente y con seguridad con los proyectos que les atraen. Tienden a ser impacientes cuando hay personas que teorizan.

Tabla 7 Estadígrafos descriptivos del desempeño académico en el área de Comunicación

\begin{tabular}{lcccccc}
\hline & \multicolumn{5}{c}{ Competencias Comunicativas } & \\
\cline { 2 - 5 } & $\begin{array}{c}\text { Comprende } \\
\text { textos orales }\end{array}$ & $\begin{array}{c}\text { Se expresa } \\
\text { oralmente }\end{array}$ & $\begin{array}{c}\text { Comprende } \\
\text { textos } \\
\text { escritos }\end{array}$ & $\begin{array}{c}\text { Produce } \\
\text { textos } \\
\text { escritos }\end{array}$ & $\begin{array}{c}\text { Interactúa con } \\
\text { expresiones } \\
\text { literarias }\end{array}$ & Promedio \\
\hline $\mathrm{N}^{\circ}$ de casos & 63 & 63 & 63 & 63 & 63 & 63 \\
Media & 13.59 & 13.57 & 14.32 & 13.71 & 13.84 & 13.81 \\
Desviación estándar & 1.881 & 1.957 & 2.488 & 2.419 & 2.573 & 1.795 \\
Coef. de variabilidad & $13.8 \%$ & $14.4 \%$ & $17.4 \%$ & $17.6 \%$ & $18.6 \%$ & $13.0 \%$ \\
Varianza & 3.537 & 3.829 & 6.188 & 5.853 & 6.620 & 3.221 \\
Mínimo & 11 & 10 & 8 & 7 & 7 & 10 \\
Máximo & 18 & 18 & 18 & 17 & 17 & 17 \\
\hline
\end{tabular}

Fuente: Reporte de Notas Registradas - Trimestre I - 2017 
En la tabla 7 se presenta los resultados de los estadígrafos descriptivos del desempeño académico en el área de Comunicación de las estudiantes. Ello permite analizar y jerarquizar los datos de las competencias del área de comunicación.

En $1^{\circ}$ lugar se ubica la competencia Comprende textos escritos, por obtener una media de 14.32 puntos y una desviación estándar de 2.488, en un rango que va desde 8 puntos como mínimo hasta 18, como máximo. Asimismo, presenta un coeficiente de variabilidad de $17.4 \%$, lo cual indica que sus datos son regularmente variables. En $2^{\circ}$ lugar se sitúa la competencia Interactúa con expresiones literarias, por lograr una media de 13.84 puntos y una desviación estándar de 2.573 , en un rango que va desde 7 puntos como mínimo hasta 17, como máximo. Además, su coeficiente variabilidad de $18.6 \%$, indica que sus datos son regularmente variables. En $3^{\circ}$ lugar se encuentra la competencia Produce textos escritos, por conseguir una media de 13.71 puntos y una desviación estándar de 2.419, en un rango que va desde 7 puntos como mínimo hasta 17, como máximo. Asimismo, presenta un coeficiente variabilidad de $17.6 \%$, con datos regularmente variables. En $4^{\circ}$ lugar está la competencia Comprende textos orales, por tener una media de 13.59 puntos y una desviación estándar de 1.881, en un rango que va desde 11 puntos como mínimo hasta 18, como máximo. Asimismo, su coeficiente de variabilidad de $13.8 \%$, sus datos son regularmente homogéneos. El $5^{\circ}$ lugar lo ocupa la competencia Se expresa oralmente, por obtener una media de 13.57 puntos y una desviación estándar de 1.957, en un rango que va desde 10 como puntaje mínimo hasta 18 como puntaje máximo. Además, tiene un coeficiente de variabilidad de $14.4 \%$, lo cual explica que sus datos son regularmente homogéneos. Concerniente al Promedio del área de Comunicación, la muestra alcanzó una media de 13.81 puntos y una desviación estándar de 1.795, en un rango que va desde 10 puntos como mínimo hasta 17, como máximo. Además, presenta un coeficiente variabilidad de 13\%, lo cual indica que los datos son regularmente homogéneos. Partiendo de estos datos, se puede señalar que las estudiantes destacaron más en la competencia Comprende textos escritos (14.32), pero tuvieron menor desarrollo o progreso en la competencia Se expresa oralmente (13.57). De modo general, respecto del desempeño académico de las estudiantes en el área de Comunicación, se sostiene que alcanzaron un puntaje $(13,81)$ correspondiente al nivel logro esperado. Esto significa que la mayoría no lograron desarrollar cabalmente las competencias comunicativas, por lo que se debe poner énfasis en la selección de los estilos de aprendizaje.

Tabla 8 Nivel de rendimiento académico en el área de Comunicación

\begin{tabular}{|c|c|c|c|c|c|c|c|c|c|c|}
\hline \multirow{3}{*}{$\begin{array}{c}\text { Rendimiento } \\
\text { Académico en el } \\
\text { Área de } \\
\text { Comunicación }\end{array}$} & \multicolumn{8}{|c|}{ Niveles de Logro } & \multirow{2}{*}{\multicolumn{2}{|c|}{ Total }} \\
\hline & \multicolumn{2}{|c|}{$\begin{array}{c}\text { En } \\
\text { Inicio } \\
(00-10)\end{array}$} & \multicolumn{2}{|c|}{$\begin{array}{c}\text { En } \\
\text { Proceso } \\
(11-13) \\
\end{array}$} & \multicolumn{2}{|c|}{$\begin{array}{c}\text { Logro } \\
\text { Esperado } \\
(14-17) \\
\end{array}$} & \multicolumn{2}{|c|}{$\begin{array}{c}\text { Logro } \\
\text { Destacado } \\
(18-20) \\
\end{array}$} & & \\
\hline & fi & $\%$ & fi & $\%$ & fi & $\%$ & fi & $\%$ & fi & $\%$ \\
\hline Promedio & 2 & 3.2 & 22 & 34.9 & 39 & 61.9 & 0 & 0.0 & 63 & 100 \\
\hline
\end{tabular}

Fuente: Reporte de Notas Registradas - Trimestre I - 2017 
RENDIMIENTO ACADÉMICO EN EL ÁREA DE COMUNICACIÓN

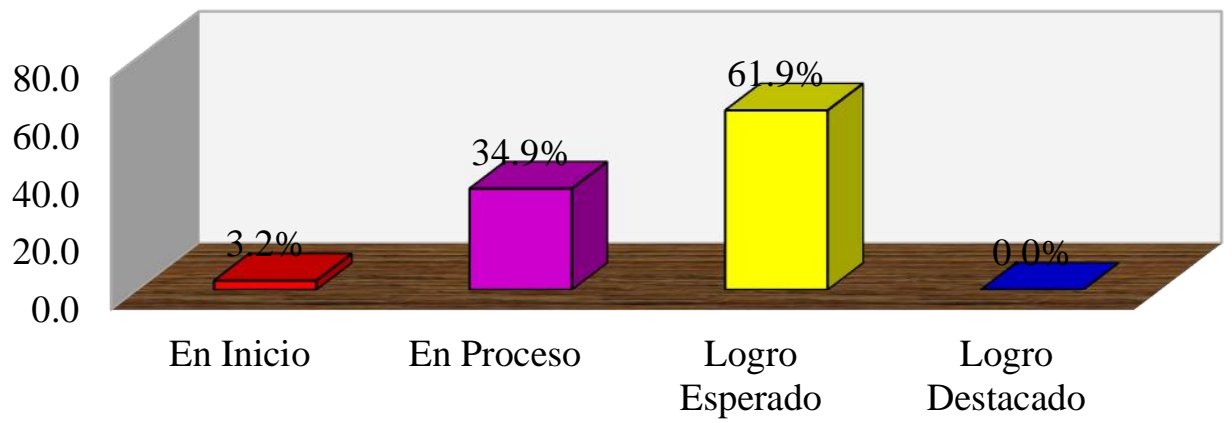

Figura 2 Nivel de rendimiento académico en el área de Comunicación

En la tabla 8 y figura 2 se muestra los resultados del nivel de desempeño académico en el área de Comunicación de las estudiantes. Se puede observar que del 100\% (63) de las estudiantes, el 61.9\% se encuentran en el nivel logro esperado, lo cual significa que ellas evidenciaron el nivel esperado en el desarrollo de las competencias comunicativas, demostrando manejo satisfactorio en todas las tareas propuestas y en el tiempo programado. Por otra parte, el 34.9\% se ubican en el nivel en proceso, lo cual indica que este porcentaje considerable de estudiantes están próximos al nivel esperado respecto a las competencias, por lo que es menester el acompañamiento durante un tiempo razonable para que logren los aprendizajes esperados. Además, el 3.2\% de estudiantes se sitúan dentro del nivel en inicio, aunque en menor porcentaje, pero estas estudiantes muestran un progreso mínimo en las competencias comunicativas de acuerdo con el nivel esperado y evidencian con frecuencia dificultades en el desarrollo de las tareas, por lo que necesitan mayor tiempo de acompañamiento e intervención del docente. Por último, el (0.0\%) de estudiantes alcanzó el nivel logro destacado, es decir, nadie evidenció un nivel superior a lo esperado respecto a las competencias comunicativas.

Tabla 9 Correlación entre los estilos de aprendizaje y el rendimiento académico en el área de Comunicación

\begin{tabular}{llc}
\hline & & Promedio \\
\hline \multirow{2}{*}{ Estilo de Aprendizaje } & Correlación de Pearson & .189 \\
Activo & Sig. (bilateral) & .138 \\
Estilo de Aprendizaje & $\mathrm{N}$ & 63 \\
Reflexivo & Correlación de Pearson &, $489^{* *}$ \\
& Sig. (bilateral) & .000 \\
Estilo de Aprendizaje Teórico & $\mathrm{N}$ & 63 \\
& Correlación de Pearson &, $353^{* *}$ \\
& Sig. (bilateral) & .005 \\
Estilo de Aprendizaje Pragmático & $\mathrm{N}$ & 63 \\
& Correlación de Pearson &, $292^{*}$ \\
& Sig. (bilateral) & .020 \\
\hline
\end{tabular}

**. La correlación es significativa en el nivel 0,01 (2 colas).

*. La correlación es significativa en el nivel 0,05 ( 2 colas).

Fuente: Cuestionario de Honey - Alonso de Estilos de Aprendizaje (CHAEA) y Reporte de Notas Registradas Trimestre I - 2017

En la tabla 9 se muestra los resultados de la relación existente entre los estilos de aprendizaje (activo, reflexivo, teórico, pragmático) y el desempeño académico en el área de Comunicación de las estudiantes. De esta forma, se puede constatar que existe una relación positiva muy baja y no significativa entre el estilo 
de aprendizaje activo y el desempeño académico en el área de Comunicación, puesto que alcanzó un coeficiente de correlación de $\mathrm{r}=.189$. Al contrastar su hipótesis estadística, se aprecia una significancia bilateral de .138 , la cual es mayor a $\propto(0,05)$, por lo que se acepta la hipótesis nula $(\mathrm{Ho})=$ No existe relación significativa entre el estilo de aprendizaje activo y el rendimiento académico en el área de Comunicación de las estudiantes.

\section{DISCUSIÓN}

En esta investigación se pudo corroborar que la relación existente entre el estilo de aprendizaje reflexivo y el desempeño académico en el área de Comunicación de las estudiantes es positiva moderada ( $\mathrm{r}$ $=, 489^{* *}$ ) y significativa (en el nivel 0,01). Por otra parte, la relación existente entre los estilos de aprendizaje: teórico $(\mathrm{r}=, 353 * *)$ y pragmático $(\mathrm{r}=, 292 *)$ con el desempeño académico en el área de comunicación es positiva baja y significativa (en el nivel 0,01 y 0.05 , respectivamente), no sucediendo de igual forma entre el estilo de aprendizaje activo y el rendimiento académico en el área de comunicación, ya que existe una relación positiva muy baja $(\mathrm{r}=.189)$ y no significativa.

Estos resultados, se relacionan en parte a los hallazgos de la investigación realizada por (Manzano \& Hidalgo, 2009), cuando concluyeron que los estilos pragmático y reflexivo se asocian al uso frecuente de estrategias de lectura; y estos influyen en el desempeño académico de la lengua extranjera. Así también, Jara (2010), en su tesis doctoral, corroboró que existe una correlación significativa entre los estilos de aprendizaje reflexivo y teórico con el desempeño académico en Educación para el trabajo. Lo cierto es que, pese a estos hallazgos científicos que afirman la fuerte asociación de las dos variables, los docentes aún no han logrado familiarizarse habitualmente con los estilos de aprendizaje en su quehacer pedagógico, por lo que el desarrollo de cada estilo se sigue dando en niveles bajos, lo cual garantiza también, la permanencia de una calidad cuestionable de aprendizajes.

Por otro lado, se encontró que el estilo de aprendizaje reflexivo (13.67) predomina en las estudiantes y consecutivamente están los estilos de aprendizaje: teórico (13.14), pragmático (12.32) y activo (10.73). Además, la mayoría de las estudiantes evidencian un nivel de preferencia moderada respecto de los estilos: reflexivo (57.1\%), Activo (42.9\%) y pragmático (41.3\%); mas por el estilo teórico, la mayoría (41.3\%) evidenció un nivel de preferencia alta. Estos resultados se asemejan al estudio realizado por Calla (2012), cuando con su estudio demostró que el estilo de aprendizaje predominante en los estudiantes es el reflexivo, con un promedio de 13,5\%. Asimismo, afirmó que sobresale el nivel de preferencia moderada en los cuatro estilos: activo, reflexivo, teórico y pragmático. Por esta razón, se infiere que la mayoría de los estudiantes tienen dificultades para alcanzar los niveles de preferencia alta y muy alta en todos los estilos de aprendizaje, realidad contraria a (Honey \& Mumford, 1986) al expresar que lo ideal sería que todas las personas fueran capaces de experimentar, reflexionar, realizar hipótesis y aplicarlos con eficacia, ya que ello les permitiría aprender y adaptarse con facilidad a cualquier circunstancia de aprendizaje que les toque vivir, y consecuentemente, serían personas competentes para afrontar los retos que plantea la realidad, pero lamentablemente las personas son más capaces de una cosa que de otra. Por otra parte, es menester reflexionar sobre la media $(13,81)$ que alcanzó el promedio de nota de las estudiantes en el área de Comunicación. El mismo que se justifica con los datos de que el $61.9 \%$ de estudiantes se encuentran en el nivel logro esperado; el 34.9\%, en el nivel en proceso; el 3.2\%, dentro del nivel en inicio y ninguna alcanzó el nivel logro destacado.

En definitiva, esta realidad tiene implicancias serías sobre los problemas en la mayoría de las estudiantes, así también lo demostró Jara (2010), en una de sus conclusiones específicas, afirmando que el mayor porcentaje de los alumnos de $2^{\circ}$ de secundaria (59.4\%) se concentra en el nivel de proceso, seguido del $24.6 \%$ que se ubica en el nivel de logro, mientras que el $15.9 \%$ se encuentra en el nivel de inicio en el rendimiento académico, no encontrándose ningún alumno en el nivel de logro satisfactorio. La explicación que la autora brinda sobre esta situación se fundamenta en que ello se debe al insuficiente apoyo en el 
hogar, así como a la falta de recursos económicos, ya que muchos de los alumnos laboran después de terminar sus clases en el terminal pesquero, suscitándose así en ellos, una actitud conformista respecto a sus logros de aprendizaje. Causas que influyen en la baja calidad de aprendizaje, pero también están otros factores como: la metodología y estrategias de enseñanza - aprendizaje, la consideración de los estilos de aprendizaje en la acción educativa, la infraestructura, los hábitos de estudio, la tecnología, la motivación..., variables que han sido y deberían seguir siendo motivo de investigación para mejorar la calidad de los aprendizajes, coincidiendo con (Dunn \& Dunn, 1984; Rettis Salazar, 2016; Schmeck, 1988). Finalmente, cabe agregar que, a partir de los datos de la media de las competencias comunicativas de las estudiantes, se determinó que ellas destacaron más en la competencia Comprende textos escritos (14.32) y presentaron debilidades en la competencia Se expresa oralmente (13.84). Por lo tanto, ante estos resultados se evidencia que no se lograron los desempeños académicos, calificativos deficientes (Parra et al., 2014), es necesario trabajar en los hábitos de estudio por lo que constituyen una estrategia de aprendizaje relevante para mejorar el rendimiento académico de los estudiantes (Gaeta et al., 2016; Suárez et al., 2012).

\section{CONCLUSIONES}

La relación existente entre el estilo de aprendizaje reflexivo y el desempeño académico en el área de Comunicación de las estudiantes del cuarto grado de la Institución Educativa Secundaria "Carlos Rubina Burgos" de Puno, 2017, es positiva moderada $\left(\mathrm{r}=, 489^{* *}\right)$ y significativa (en el nivel 0,01$)$. Por otra parte, la relación existente entre los estilos de aprendizaje: teórico $(r=, 353 * *)$ y pragmático $(r=, 292 *)$ con el desempeño académico en el área de comunicación es positiva baja y significativa (en el nivel 0,01 y 0.05, respectivamente), no ocurriendo de la misma forma entre el estilo de aprendizaje activo y el rendimiento académico en el área de comunicación, ya que existe una relación positiva muy baja $(r=.189)$ y no significativa. Las estudiantes evidencian un nivel de preferencia moderada respecto de los estilos de aprendizaje: reflexivo (57.1\%), activo (42.9\%) y pragmático (41.3\%); sin embargo, respecto del estilo de aprendizaje teórico (41.3\%) evidencian un nivel de preferencia alta. Las estudiantes, en cuanto a su rendimiento académico en el área de comunicación, se encuentran en el nivel logro esperado en un $61.9 \%$. Además, esto se reafirma con el resultado de la media de las notas de las estudiantes, que dio un puntaje de 13,81 puntos, lo cual indica que la mayoría alcanzó con dificultades el nivel logro esperado.

\section{REFERENCIAS BIBLIOGRÁFICAS}

Alonso, C. (1992). Análisis y diagnóstico de los estilos de aprendizaje en estudiantes universitarios. Retrieved January 10, 2019, from https://www.researchgate.net/publication/39132740_Analisis_y_diagnostico_de_los_estilos_de_apr endizaje_en_estudiantes_universitarios

Alonso, C., Gallego, D., \& Honey, P. (1994). Los estilos de aprendizaje. Bilbao. Retrieved from https://www2.uned.es/revistaestilosdeaprendizaje/numero_1/resena_alonso-gallego_honey_es.pdf

Alonso, C., Gallego, D., \& Honey, P. (2007). (PDF) Los Estilos de Aprendizaje: Procedimientos de diagnóstico y mejora,. (Editorial Mensajero, Ed.) $\left(7^{\circ}\right)$. Bilbao. Retrieved from https://www.researchgate.net/publication/311452891_Los_Estilos_de_Aprendizaje_Procedimientos _de_diagnostico_y_mejora

Aragon de Viau, M. (2000). Estilos de Aprendizaje. Guatemala. Retrieved from http://biblio3.url.edu.gt/Libros/2011/est_aprende.pdf

Biggs, J. (2006). Calidad del aprendizaje universitario (NARCEA, S.). Madrid.

Cachay, S. (2014). Estilos de aprendizaje y rendimiento académico en estudiantes de la EAP de Administración De La Universidad Peruana Unión, 2014. Universidad Peruana Unión Escuela de Posgrado Unidad de Posgrado en Educación. Retrieved from https://core.ac.uk/download/pdf/54242623.pdf

Carrasco, S. (2005). Metodología de la Investigación Científica. Lima: Lima, Universidad Nacional de San 


\section{Marcos.}

Coloma, C., Manrique, L., Revilla, D., \& Tafur, R. (2008). (PDF) Estudio descriptivo de los estilos de aprendizaje de docentes universitarios. Revista Estilos de Aprendizaje, 1, 1-22. Retrieved from https://www.researchgate.net/publication/28290185_Estudio_descriptivo_de_los_estilos_de_aprendi zaje_de_docentes_universitarios

De Murcia, U., Fernández, E., Bernardo, E. ;, Suárez, A. ; Cerezo, N. ; Núñez, R. ; \& Rosário, J. C. ; (2013). Predicción del uso de estrategias de autorregulación en educación superior. Anales de Psicología, 29, 865-875. https://doi.org/10.6018/analesps.29.3.139341

Diaz, A. (2010). La Motivación y los estilos de aprendizaje y su influencia en el nivel de rendimiento académico de los alumnos de primer a cuarto año en el área del idioma inglés de la Escuela de Oficiales de la FAP. Universidad nacional Mayor de San Marcos, Lima.

Dunn, R., \& Dunn, K. (1984). Modelo Dunn y Dunn. Retrieved December 18, 2018, from http://www.cca.org.mx/profesores/cursos/cep21/modulo_2/modelo_dunn_dunn.htm

Esteban, M., \& Ruiz, C. (1996). Estilos y estrategias de aprendizaje Coordinadores (Vol. 12). Retrieved from https://digitum.um.es/jspui/bitstream/10201/10118/1/Estilos y estrategias de aprendizaje.pdf

Farias, F., Díaz, M., \& Miranda, F. (2012). Estilos de aprendizaje y su relación con el rendimiento escolar en la enseñanza media. Estilos de Aprendizaje: Investigaciones y Experiencias: [V Congreso Mundial de Estilos de Aprendizaje], Santander, 27, 28 y 29 de Junio de 2012, 2012, ISBN 978-84-695-34540 . Retrieved from https://dialnet.unirioja.es/servlet/articulo?codigo $=4643232$

Gaeta, Martha Leticia; Teruel, M. Pilar; Orejudo, S. (2012). ERIC - Motivational, Volitional and Metacognitive Aspects of Self Regulated Learning, Electronic Journal of Research in Educational Psychology, 2012. Retrieved January 19, 2019, from https://eric.ed.gov/?id=EJ970379

Gaeta, M., Cavazos, J., \& Arroyo, C. (2016). CPU-e, Revista de Investigación Educativa, 23, 142-166. Retrieved from http://www.redalyc.org/articulo.oa?id=283146484008

Gallego, D., \& Alonso, C. (2007). Los estilos de aprendizaje como una estrategia pedagógica del Siglo XXI Learning Styles As A Teaching Strategy Of The XXI Century. Revista Electrónica de Socioeconomía Estadística e Informática, 1, 20-41. Retrieved from http://www.cm.colpos.mx/revistaisei/numeros/RESEI_N1V1_020.pdf

Garay, L. (2015). Estilos de aprendizaje e inteligencias múltiples en estudiantes universitarios. LIMA. 2014. Retrieved from http://www.repositorioacademico.usmp.edu.pe/bitstream/usmp/1967/1/garay_ple.pdf

García Cue, J. L. (2006). Los estilos de aprendizaje y las tecnologías de la información y la comunicación en la formación del profesorado. UNED. Universidad Nacional de Educación a Distancia (España). Retrieved from https://dialnet.unirioja.es/servlet/tesis?codigo=38926

García, J., Sanchez, C., Gutierrez, M., \& Jimenez, M. (2012). Estilos de Aprendizaje y Estrategias de Aprendizaje: un estudio en discentes de postgrado. Review of Learning Styles, 10, 2012. Retrieved from http://www.uned.es/congreso-estilos-aprendizaje/

García, J., Santizo, J., \& Alonso, C. (2009). Uso de las TIC de acuerdo a los estilos de aprendizaje de docentes y discentes. Revista Iberoamericana de Educación, 48, 2-10. Retrieved from https://www.researchgate.net/publication/28237719_Uso_de_las_TIC_de_acuerdo_a_los_estilos_de _aprendizaje_de_docentes_y_discentes/download

Gregorc, A. (1982). An adult's guide to style. [Maynard MA]: [Gabriel Systems Inc.]. Retrieved from https://www.worldcat.org/title/adults-guide-to-style/oclc/9956826?referer=di\&ht=edition

Gutiérrez, M. (2018). Estilos de aprendizaje, estrategias para enseñar. Su relación con el desarrollo emocional y "aprender a aprender." Tendencias Pedagógicas, 31(2018), 83-96. https://doi.org/10.15366/tp2018.31.004

Honey, P., \& Mumford, A. (1986). Modelo de Honey y Mumford. Retrieved January 10, 2019, from http://www.cca.org.mx/profesores/cursos/cep21/modulo_2/mod_honey_mumford.htm

Jara, G. (2010). "Estilos de aprendizaje y rendimiento académico de estudiantes de $2^{\circ}$ de secundaria en educación para el trabajo de una institución educativa del Callao". Universidad San Ignacio de Loyola. Retrieved from http://repositorio.usil.edu.pe/bitstream/123456789/1190/1/2010_Jara_Estilos de aprendizaje y rendimiento académico de estudiantes de $2^{\circ}$ de secundaria en educación para el trabajo.pdf

Jimenez, M. (2000). Competencia social: intervención preventiva en la escuela. Infancia y Sociedad, 24, $21-48$. 
Keefe, J., \& Ferrel, B. (1990). Developing a defensible Learning Style Paradigm. Retrieved from https://pdfs.semanticscholar.org/f9f5/42869986f4a369251ef3c96a9e6d98fd29be.pdf

Manzano, M., \& Hidalgo, E. (2009). Estilos de aprendizaje, estrategias de lectura y su relación con el rendimiento académico de la lengua extranjera. Educación XXI, 12, 123-150. Retrieved from http://www.redalyc.org/articulo.oa?id=70611919007

Parra, J., Cerda, C., López, O., \& Saiz, J. (2014). Género, autodirección del aprendizaje y desempeño académico en estudiantes de pedagogía. Educación y Educadores, 17(1), 91-107. https://doi.org/10.5294/edu.2014.17.1.5

Pozo, J., Monereo, C., \& Castelló, M. (2001). El uso estratégico del conocimiento. Psicología de La Educación Escolar, $\quad 8, \quad 211-234 . \quad$ Retrieved from https://www.researchgate.net/publication/261082131_EL_USO_ESTRATEGICO_DEL_CONOCI MIENTO

Rettis Salazar, H. T. (2016). Estilos de aprendizaje y rendimiento académico de la asignatura de estadística de los estudiantes del III ciclo de la EAPA, Facultad de Ciencias Administrativas - UNMSM - 2015. Repositorio de Tesis - UNMSM. Universidad Nacional Mayor de San Marcos. Retrieved from http://cybertesis.unmsm.edu.pe/handle/cybertesis/4780

Rojas, G., Salas, R., \& Jimenez, C. (2006). Estilos de aprendizaje y estilos de pensamiento entre estudiantes universitarios. Estudios Pedagógicos (Valdivia), 32(1), 49-75. https://doi.org/10.4067/S071807052006000100004

Rosário, P., Rosario, P., Mourão, R., Baldaque, M., Nunes, T., Nuñez, J. C., ... Valle, A. (2009). Volumen 14. No 2. Págs. Retrieved from https://www.redalyc.org/pdf/175/17512724002.pdf

Schmeck, R. (1988). Learning Strategies and Learning Styles (New York:).

Suárez, N., Fernández, E., Cerezo, R., Rodríguez, C., Rosário, P., \& Núñez, J. C. (2012). Tareas para casa, implicación familiar y rendimiento académico. Aula Abierta, 40(1), 73-84. Retrieved from https://core.ac.uk/download/pdf/71849378.pdf

Yana, M., \& Adco, H. (2018). Acompañamiento pedagógico y el rol docente en jornada escolar completa: Caso instituciones educativas Santa Rosa y Salesianos de San Juan Bosco - Puno Perú. Revista de Investigaciones Altoandinas - Journal of High Andean Research, 20(1), 137-148. https://doi.org/10.18271/ria.2018.337

Zimmerman, B. J. (1989). Title: A Social Cognitive View of Self-Regulated Academic. Journal of Educational Psychology (Vol. 81). Retrieved from http://web2.epnet.com.proxy.lib.sfu.ca/citation.asp?tb=1\&_ug=sid+AE515792-89... 